\title{
Analysis on Interactive Stage Rehearsal on Stage
}

\author{
Yang Chen \\ Huanghe Science and Technology College \\ Zhengzhou, China
}

\begin{abstract}
Aiming at the specific demand of enhancing the visual expression of stage, with 3D visualization engine as the platform, the interactive stage lighting rehearsal system realizes the control of functional modules of stage such as the lighting projection pattern, the rotation angle, the switching color, the adjusting beam angle, linear dimming and spatial positioning through virtual visual presentation and human-computer interaction, and develops a unified data interface, builds the collaborative control module of the stage lighting visual effects, and achieves technological innovation of the stage lighting rehearsal.
\end{abstract}

Keywords-simulation engine; human-computer interaction; lighting rehearsal; cooperative control

\section{INTRODUCTION}

Stage lighting, also known as "stage illumination", is one of the means of stage art modeling. Stage lighting is an important part of the performance space, an art creation which implements the all-round lighting design of the visual environment based on specific circumstances required by the development of the plot of the characters, and reproduces the design intent in the form visual image to the audience on purpose.

In modern society, stage performances are increasingly inseparable from the support of stage lighting. The stage lighting is daedal and the projected beams are rich and colorful, which gives the audience new, unique and even shocking visual effects. Due to the variability of the stage lighting and the good effect it brings to stage performance, the stage program directors have been paying more and more attention to the application of stage lighting. The stage lighting performance effect has become an important basis for judging the success of some large stage performances.

However, there are still many places that need to be improved in the traditional lighting rehearsal system. For example, the study on the stage lighting system still remains in the aspects of hardware implementation of a single lamp or data communication, and study on the theory modeling of the entire stage lighting system and collaborative control is still lacked. The stage lighting on the site is mostly adjusted through the lighting engineer's observation as a feedback to the effect in real time, which significantly increases the workload of stage creative staff. Because of the different characteristics and shortcomings of existing control systems or methods, it is necessary to find a rehearsal framework and control method for the entire stage lighting system.
In order to solve the above problems, around the specific demand of strengthening the stage visual performance, the interactive stage lighting rehearsal system takes 3D simulation engine as the platform, realizes the control of functional modules of stage such as the lighting projection pattern, the rotation angle, the switching color, the adjusting beam angle, linear dimming and spatial positioning through virtual visual presentation and human-computer interaction, and develops a unified data interface, builds the collaborative control module of the stage lighting visual effects, and achieves technological innovation of the stage lighting rehearsal.

\section{SYSTEM FEATURES}

The interactive stage lighting rehearsal system makes full use of computer's simulation technology, real-time rendering technology and human-computer interaction technology.

The system takes 3D simulation engine's data processing module as the core, realizes the selection and adjustment of the stage lighting's type and attribute through the interactive console, realizes the real-time control of the lighting through the external equipment control and parameter setting, transfers the data to the stage machinery according to the unified data interface, and realizes the collaborative control of the simulation system and the stage machinery. The system can realize the non-destructive output of the frame sequence with the $2 \mathrm{D} / 3 \mathrm{D}$ modes according to the stage creativity or postproduction demand, and realize the recording and playback of the stage lighting rehearsal effect.

The system can perform stage lighting comprehensive rehearsal before the formal performance, provides visual stage lighting effects and basis the formal performance, reduces the human and material resources and time costs, avoids waste of resources and improves efficiency.

\section{SYSTEM ARCHITECTURE}

The interactive stage lighting rehearsal system includes: interactive control module, data solving module, output module. "Fig.1"

The interactive control module uses UI interactive control platform to make software and create screen UI console. By importing the readable specific format file of the engine which describes the UI control platform into the $3 \mathrm{D}$ simulation engine, it can build a UI interactive control platform in the engine. The UI control node is created by code realization and describes the logical relationship between it and the external controller device, so as to realize the operation of UI console 
of the screen; the multiple UI drop-down menu contains all types of stage lighting, such as: conventional stage lighting, computer stage lighting and LED stage lighting. The attribute of the light that has been loaded on the stage can be changed in the drop-down menu in UI interactive platform after being selected by writing the attribute transformation node, and binding the node on the button and selecting the node to be replaced.



Fig. 1. System architecture

The parameters of stage lights need to be controlled after the stage lights are loaded, and the control methods can be divided into two types: one is the external control method, suitable for lighting effect requiring precise control, which accurately sets the parameters information through keyboard, handheld devices such as rocker or other professional control equipment, and completes actions such as the rotation of stage lights and elevation; the other is the data-driven method, suitable for stage play, concert, party and so on based on datadriven method, which needs to watch the overall effect of program from beginning to end, and can play back and adjust the parameter information. In addition, the parameter information adjusted with external control method can be programmed as preset-format text to be read by the data-driven module.

Data processing module refers to the description of the changing process of various parameters of the stage lighting by 3D simulation engine. The interaction between the external control device and 3D graphic simulation engine can be realized by registering and initializing the external control device in 3D graphic simulation engine. Each time the system is updated, it calls the input of function and the listener function, it can monitor and solve the data of each axis of the control device. Add mechanism to prevent error in the control of the stage lighting by external control equipment. Turn the critical value of the external input device data to the critical section, and the values other than the critical section correspond to the original interval through interpolation. The optimization of the data controlled by external input device can reduce the sensitivity of the input device in order to achieve precise control of the stage lighting. Turn the internal and external parameters of the stage lighting into the change of common variables such as the color, beam angle, brightness and position, and renders the changing effect of stage lighting through 3D simulation engine in real time.

The output module consists of 2D / 3D rendering frame sequence and mechanical control. The rendering frame sequence is to render the rehearsal effect of stage lighting by $3 \mathrm{D}$ simulation engine in real time, and 2D / 3D modes can be used to realize non-destructive output. The mechanical control part of lighting defines the data format such as the angle, color, beam angle, brightness and position of the light through the unified data interface, which realizes the makes the data docking between the simulation system and the stage machinery, and directly drives the stage lighting machinery through the lighting rehearsal data obtained through the interactive control module. That is, the stage creative staffs greatly shorten their working hours and improve work efficiency.

\section{TECHNOLOGICAL IMPLEMENTATION}

\section{A. UI interface}

The interactive console creates a screen UI control interface through Adobe Flash. The button images need to be made in the image processing software and imported into Adobe Flash, and all the button effects such as menu opening, hiding, button effect, selecting effect, drop-down menu, sliding effect of the small slider on the dragging bar are all realized by Action script code which directly writes the action code. The first-level menu of the stage lighting is shown in "Fig. 2": 


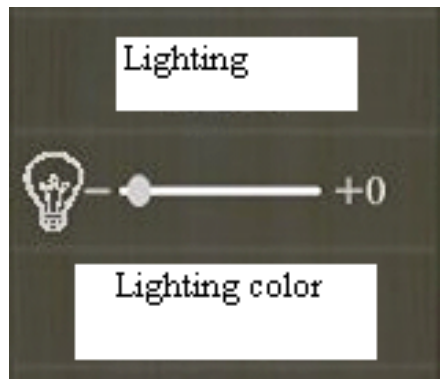

Fig. 2. First-level menu

The secondary menu can be opened by clicking the corresponding button in the first-level menu. The effect of the drop-down menu in this part is written by AS in the action instruction, and the corresponding XML file generated is read by $3 \mathrm{D}$ simulation engine. The name, the mouse effect, the cursor of the UI interaction console that need to be defined in XML, the location that needs to be displayed on UI interface, and the locking of the model in the image and model base. The secondary menu of the stage lighting is shown in "Fig. 3":

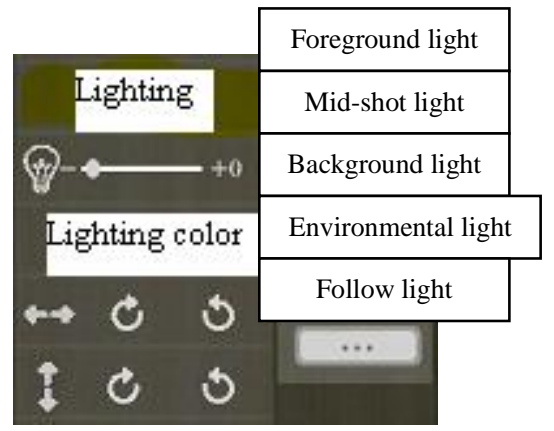

Fig. 3. Secondary menu

The effect of the secondary menu of the stage lighting is shown in "Fig. 4", the red, green and blue components in lighting can be adjusted by adjusting the red slider, green slider, blue slider to adjust the color of mixed light.

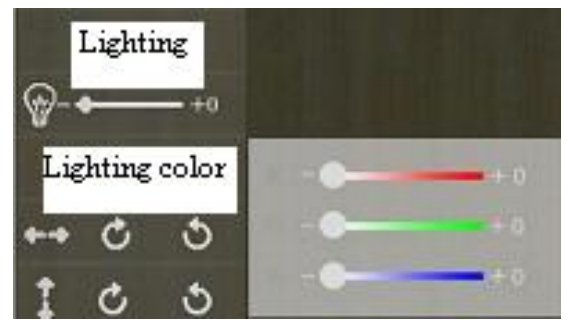

Fig. 4. Secondary menu of stage lighting color.

\section{B. Realization of Lighting Control}

In $3 \mathrm{D}$ engine, the posture of the object is presented by the Euler angle, the control of the entity rotation can be achieved through the adjustment of Euler angle of each frame. In the rotation control node, the velocity to achieve the rotation of entity is represented by $3 \mathrm{D}$ vector quantity $(\mathrm{x}, \mathrm{y}, \mathrm{z})$, in which $\mathrm{x}$ represents the rotation along the $\mathrm{X}$-axis of the entity's own coordinate system, y represents the rotation along the Y-axis of the entity's own coordinate system, and $\mathrm{z}$ represents the rotation along the Z-axis of the entity's own coordinate system. For object that need to keep rotating, it rotates at a certain speed until the state changes, for entities that need to rotate to the target posture, there are two ways to achieve, one is to move to the target posture at a certain speed, and the other is rotate to the target posture within the specified time. The difference between these two methods: the first selects and rotates the light completely through interactive operation; while the second sets the specific rotation angle of the specific light at the specific time in advance in the flow chart. That is, the first is rotation without parameters, and the second is rotation with parameters. Both the two methods are realized by the flow chart and need to write two kinds of rotating nodes, trigger the trigger node by the button, after triggering the node, connect the rotating node that has been written, so as to achieve the rotation of lighting through the button adjustment.

The light brightness transformation is realized by adjusting the sliders on the light slider on the UI interface. Firstly, select the light type that needs to be adjusted in the light menu, then adjust the brightness of the light through the sliders on the brightness slider. The adjustment of this part is achieved by binding the flow chart, to bind various types of lighting trigger node to the lighting button in secondary lighting menu, once it's selected the light node is triggered. Connect the brightness node after triggering the node, at the same time, the brightness slider binds the brightness node, adjust the brightness with the small slider, and thus achieve the brightness conversion of light.

The class diagram of the lighting control part is shown in "Fig. 5": 


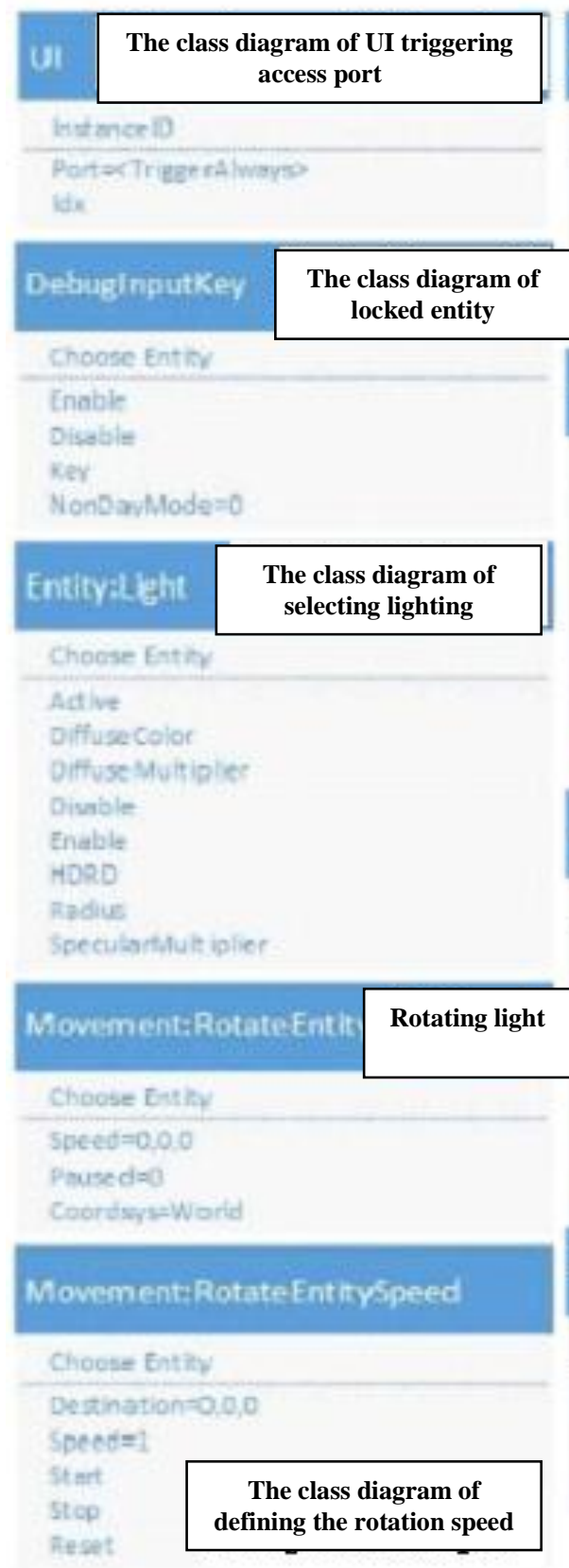

Fig. 5. The class diagram of stage lighting control

The lighting color transformation is realized by adjusting the slider on the light slider on UI interface. Firstly, select the light type that needs to be adjusted in the light menu, after selecting, adjust the brightness of the light through the slider on the brightness slider. This part of the adjustment is achieved by binding the flow chart, to bind various types of lighting trigger nodes to the lighting button in secondary lighting menu, once it's selected the light node is triggered. Because each color can be decomposed into red, green, and blue, the light color adjustment adjusts the color of a variety of lights through RGB color system. After triggering the node, connect RGB input port, namely the diffuse Color access, whose data type is $(\mathrm{R}, \mathrm{G}, \mathrm{B})$ type, at the same time, the color slider is bound to
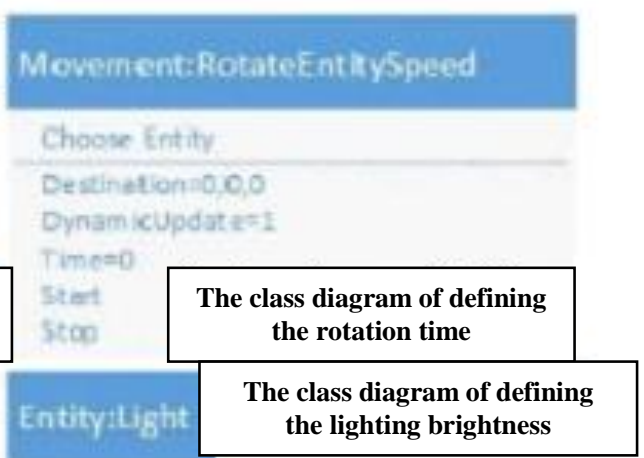

Choose Entit

Active

Diffuse Colar

DifluseMutipliet

Disable

Ensole

HoHD

Radius

Sotoulamultipliet



\section{Choose Entity \\ Antive \\ Diflusecolor \\ Diffuse hlut iplier \\ Disatis \\ HDRD \\ Radius \\ Soccula mutiplier}

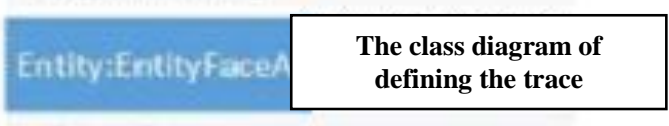

Chooue Entity

Target

porma. 0

Artivate

Deactivate

RGB entry, adjust the three colors of red, green and blue with RGB small slider to get different colors of light.

For the follow light, it is necessary to always aim at the object, which requires the orientation control of the entity. The orientation control determines the orientation vector Dir = Ptarget-Pres via the target position Ptarget and its own position Pres, set the orientation of the entity as normalized direction vector Dirnorm. In order to achieve the tracing positioning, it needs to write the flow graph node in $\mathrm{C}++$, which contains the physical coordinates and complete target positioning. The light directly aims at the entity to be positioned through control and complete the tracing and positioning of the light. 


\section{APPLICATION CASES}

\section{A. Application Stage Lighting Rehearsal of the} Opera"Ziegfried"

"Fig. 6", "Fig. 7", "Fig. 8", "Fig. 9", "Fig. 10" shows the application of the interactive stage lighting rehearsal system in the rehearsal process of the opera "Siegfried". "Fig. 6" is the effect with no lighting adjustment, "Fig. 7" is the control effect of mid-shot lighting on the stage, "Fig. 8" is the control effect of background lighting on the stage, "Fig. 9" is the control effect of global lighting on the stage, "Fig. 10" is the control effect of the color of stage computer lighting.

In order to meet the need of the development of the opera story, the different location and brightness of lighting will also change, set off and render the development trend of the story and the inner world of the protagonists.

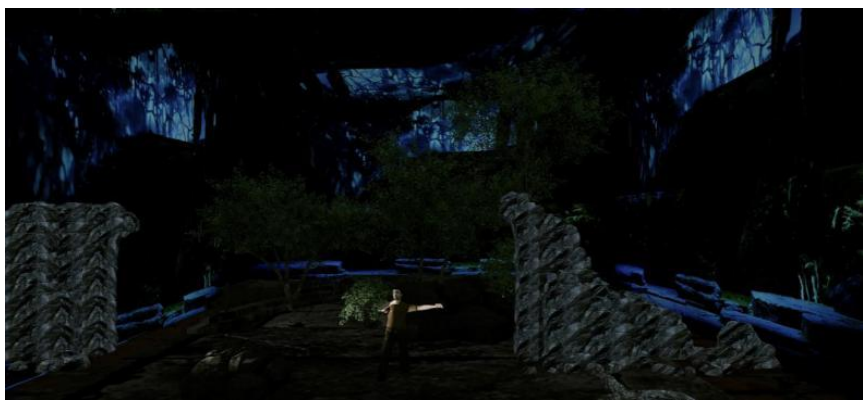

Fig. 6. No lighting effect

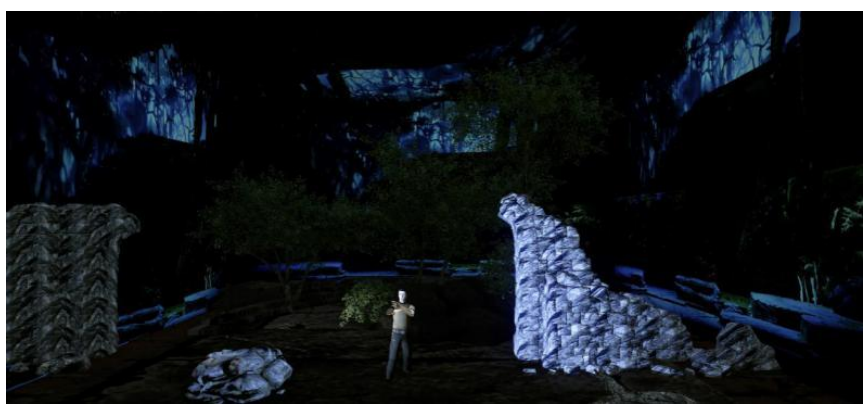

Fig. 7. Control effect of mid-shot lighting


Fig. 9. Control effect of global lighting

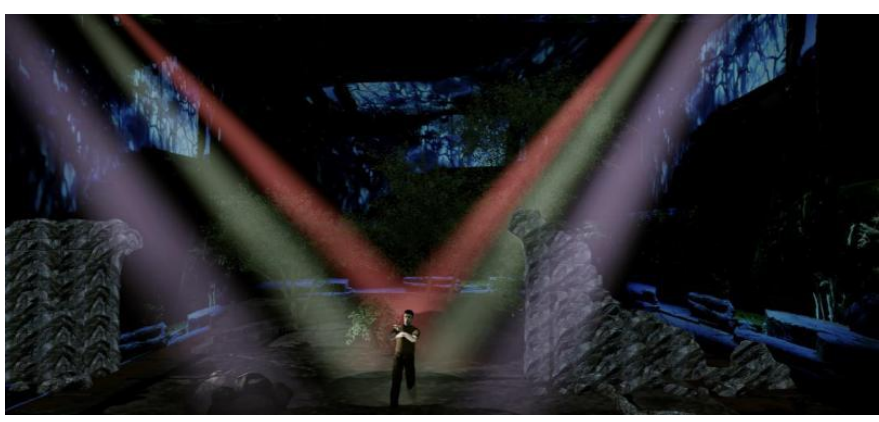

Fig. 10. The control effect of the computer lighting

\section{B. Stage Creative Application in 2014 CCTV Spring Festival Evening Gala}

"Fig. 11", "Fig. 12", "Fig. 13", "Fig. 14" show the application of interactive stage lighting rehearsal system in the stage creative rehearsal of 2014 CCTV Spring Festival Evening Gala. "Fig. 11" is effect diagram of the overall stage with no stage lighting but only LED screen video, "Fig. 12" is the control effect stage of the movement of the stage computer light, "Fig. 13" is the effect of dynamic switch and light color synchronization of the computer light, "Fig. 14" is the control effect of computer light color.

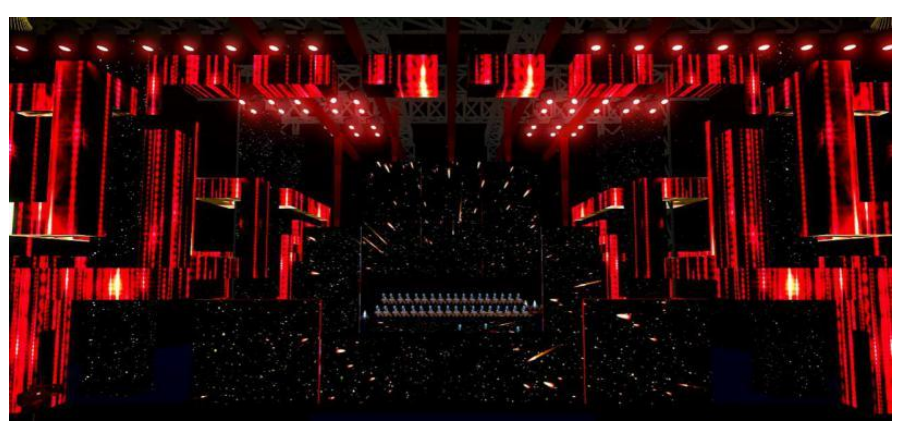

Fig. 11. Effect with no lighting

Fig. 8. Control effect of background lighting 


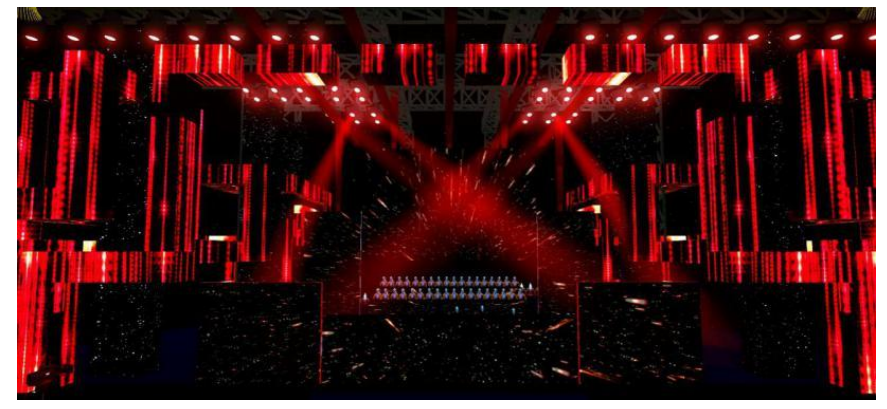

Fig. 12. Effect of the computer light adjustment

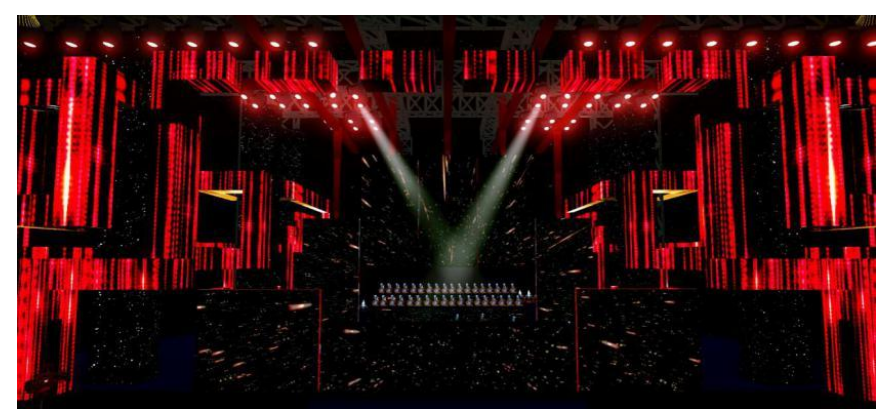

Fig. 13. Effect of the computer lighting adjustment



Fig. 14. Effect of computer lighting adjustment

\section{CONCLUSION}

Compared with the existing technology, the system has raised the stage creative arrangement and machinery collaborative control to a new level, thus truly embodies the practicability and the scientificity of the concept of digital performance, laying reliable technical support and a profound guiding significance for the simulation research of the stage performance in the future, and the system has a wide application prospect and business value in the field of future stage performance.

\section{REFERENCES}

[1] Hu Miaosheng. Stage design aesthetics. Shanghai Literature and Art Publishing House. 2002.

[2] Sheng Min, Hu Renrong, Huang Xuetong. Application and Prospect of Virtual Reality Technology in Stage Control. Construction of Venues.

[3] Wang Kaixuan, Ding Gangyi. Design and Simulation of Mechanical Movement of Dynamic Stage Based on Data [J]. Computer Simulation 2015-02: 275-279.
[4] Yi Yudan. Formal Beauty Elements of Stage Art Set Design. Journey Into Amazing Caves.

[5] He Xi. Unconscious Imagination of the Human-computer interaction. Frontier Technology. 2011. 